INTERNATIONAL JOURNAL OF ENVIRONMENT

Volume-9, Issue-2, 2019/20

ISSN 2091-2854

Received:30 March 2020

Revised:15August 2020

Accepted:17August 2020

\title{
DIVERSITY AND LOCAL USES OF TREE SPECIES OF NON-TIMBER FOREST PRODUCTS IN ZAMAY FOREST RESERVE IN CAMEROON
}

Souare Konsala ${ }^{1}{ }^{*}$, Froumsia Moksia ${ }^{1}$, Hamawa Yougouda ${ }^{2}$, Sassouang Evele Moctar ${ }^{1}$, Tchobsala ${ }^{1} \&$ Ibrahima Adamou ${ }^{3}$

${ }^{1}$ University of Maroua, Faculty of Science, Department of Biological Sciences, PO Box: 814, Maroua, Cameroon

${ }^{2}$ University of Maroua, National Advanced School of Engineering of Maroua, PO Box: 46, Maroua, Cameroon

${ }^{3}$ University of Ngaoundéré, Faculty of Science, PO Box: 454, Ngaoundéré, Cameroon

*Corresponding author: ksouare07@gmail.com

Forest products play an important role in supporting livelihoods in many developing countries. The study examined diversity and local uses of non-timber forest products (NTFPs) in Zamay forest reserve in Cameroon. The inventory design consisted of three collection units $(\mathrm{CU}): \mathrm{CU}_{1}$, $\mathrm{CU}_{2}$ and $\mathrm{CU}_{3}$ in the north, center and south of the reserve respectively. In each $\mathrm{CU}$, seven plots of $2500 \mathrm{~m}^{2}$ each $(50 \mathrm{~m} \times 50 \mathrm{~m})$ were established and woody individuals $(\mathrm{dbh} \geq 10 \mathrm{~cm})$ were inventoried. Local uses of NTFP were determined through semi-structured and structured interviews and plot methods were used for the inventory of the resource species. Statistical analyses were performed with Origin 6.0 and XLSTAT Softwares. A total of 745 stems was recorded, representing fifty-two species grouped in twenty-two families and thirty-seven genera. The Shannon diversity index $\left(\mathrm{H}^{\prime}\right)$ varied from 2.52 to 3.11, and Shannon evenness index (EQ) from 0.75 to 0.83. The most dominant families were Combretaceae, Burseraceae, Balanitaceae and Mimosaceae. A total of 44 useful species was recorded in five categories of uses: medicine, food, fodder, crafts and fertilizers. The most important species in terms of use values were Acacia albida Del., Anogeissus leiocarpus (DC.) Guill. \& Perr., Khaya senegalensis (Desr.) A. Juss., Balanites aegyptiaca L., Ziziphus mauritiana Lam., Tamarindus indica L. and Sclerocarya birrea (A. Rich.) Hochst. Means of frequency of citation and use values were not significantly different. Results of this survey will allow considering reforestation with valued species in the degraded parts of the reserve.

Keywords: Tree species, local uses, NTFPs, Zamay Forest Reserve, Cameroon.

DOI: http://dx.doi.org/10.3126/ije.v9i2.32685

Copyright $@ 2020$ IJE

This work is licensed under a CC BY-NC which permits use, distribution and reproduction in any medium provided the original work is properly cited and is not for commercial purposes 


\section{Introduction}

The harvesting and consumption of plant products from natural forests is known to account for a large proportion of the livelihood of people living close to such habitats (Dovie et al., 2002; Ticktin, 2005). The majority of these plant products are non-timber forest products (NTFPs), a term that encompasses biological materials used for purposes other than for commercial timber (Ndangalasi et al., 2007). NTFPs produced in tropical forests can be grouped into four categories (Ayuk et al., 1999; Dovie et al., 2002): fruits and seeds, with plant parts harvested mainly for fleshy fruit bodies, nuts and oil seed; plant exudates such as latex, resin and floral nectar; vegetative structures such as apical buds, bulbs, leaves, stems, barks and roots, and small stems, poles and sticks harvested for housing, fencing, fuelwood, and craft and furniture materials (carvings, stools). NTFPs, due to their perceived renewable nature, are viewed as a potential means to enhance rural livelihoods and conserve biodiversity in many forested regions across the world (Hegde et al., 1996; Bawa and Gadgil, 1997). In recent years, the contribution of NTFPs to alleviate poverty, particularly in the developing world, has been widely recognized (Shackletonet al. 2005; Rasul et al. 2008).

NTFPs have a high potential for forest conservation and poverty alleviation since the 1980s (De Beer and McDermott, 1989; Peters, 1990). It was argued that the usefulness of NTFPs to the local people would encourage them to sustainably manage the forest resources. The plant-level research that comprises inventories, impact studies and monitoring is necessary if plant resources are to be harvested sustainably by human populations living adjacent to protected areas in sub-Saharan Africa (Ndangalasi et al., 2007). The NTFPs can be success to reduce hunger and provide employment for local people. There are actually few statistics to build a strong case for NTFPs in Cameroon and many other countries in the world. In fact, national level data is often limited to some export species and sometimes to some endangered species. Yet, such information is crucial at the policy level in order to encourage decision makers to continue to support sustainable development and trade in such products (Belcher et al., 2005). Despite its policy relevance, and the existence of many studies describing the value chains of NTFPs, assessing their economic value including both the quantities of NTFPS sold and self-consumed at the national level is not always an easy task. One reason is that most studies analyzing such products are project, in which case having a general picture at the national level is often difficult.

The region of Zamay forest reserve in the sudano-sahelian zone of Cameroon harbors valuable forest resources including NTFPs such as Tamarindus indica L., Ziziphus mauritiana Lam. and Diospyros mespiliformis Hochst. ex A. Rich. which contribute to sustain livelihood of many people in terms of consumption and income generation. However, poor studies have been carried out in order to assess the threats on resources and implications for their sustainability. These threats were exacerbated since 2014 by the massive arrivals of about 64000 refugees from Nigeria in 2017 and the internally displaced persons fleeing the abuses of Boko-Haram (Samantha and Njikam, 2017). They are camped in the Minawaou Camp situated about seven kilometers from the reserve and sometimes get their needs such as firewood, medicines, fruits and seeds from the periphery and even inside the reserve. The study aims to i) inventory the resource species, ii)determine the local uses of the plant resources and iii)assess their vulnerability status and explore the implications for conservation and livelihoods.

\section{Materials and methods \\ Study site}

The study was carried out in Zamay forest reserve (ZFR) in the sudano-sahelian zone of Cameroon. At its creation in 1947, it covered an area of 1000 ha and lies between 
$10^{\circ} 34^{\prime} 40.80^{\prime \prime}$ to $10^{\circ} 38^{\prime} 22.20^{\prime \prime} \mathrm{N}$ and $13^{\circ} 51^{\prime} 54.00^{\prime \prime}$ to $13^{\circ} 56^{\prime} 7.80^{\prime}$ ' $\mathrm{E}$ with an average altitude of $582 \mathrm{~m}$ (Figure 1). The annual average of rainfall is $849.7 \mathrm{~mm}$ with a temperature of $26.2^{\circ} \mathrm{C}$ (Suchel, 1987). ZFR is surrounded by three mountains: the Kapsiki plateau in the west, the granite ranges of Mofou in the east and the Gadala Mountain in the north. Vertisols, tropical ferruginous soils, raw mineral soils and halomorphic soils are the most represented soils in the area where rainy season crops are grown (Téwéché et al., 2016). The plant cover is made up of a set of natural stands bringing together indigenous forest species such as Boswellia dalzielii Hutch., Isoberlinia doka Craib \& Stapf, Anogeissus leiocarpus (DC.) Guill. \& Perr., Diospyros mespiliformis Hochst ex A. Rich, Khaya senegalensis (Desr.) A. Juss., Ziziphus mauritiana Lam. and an artificial stand consisting of planted forest species such as Azadirachta indica A. Juss. and Senna siamea (Lam.) Irwin \& Barneby (Letouzey, 1985). These plants are lined with small grasses which are only used for livestock feed. Despite anthropogenic action (illegal cutting, grazing and agriculture) the density is considerable and the existing trees represent $2 / 3$ of trees at the creation of the said perimeter even as the area has decreased by half.

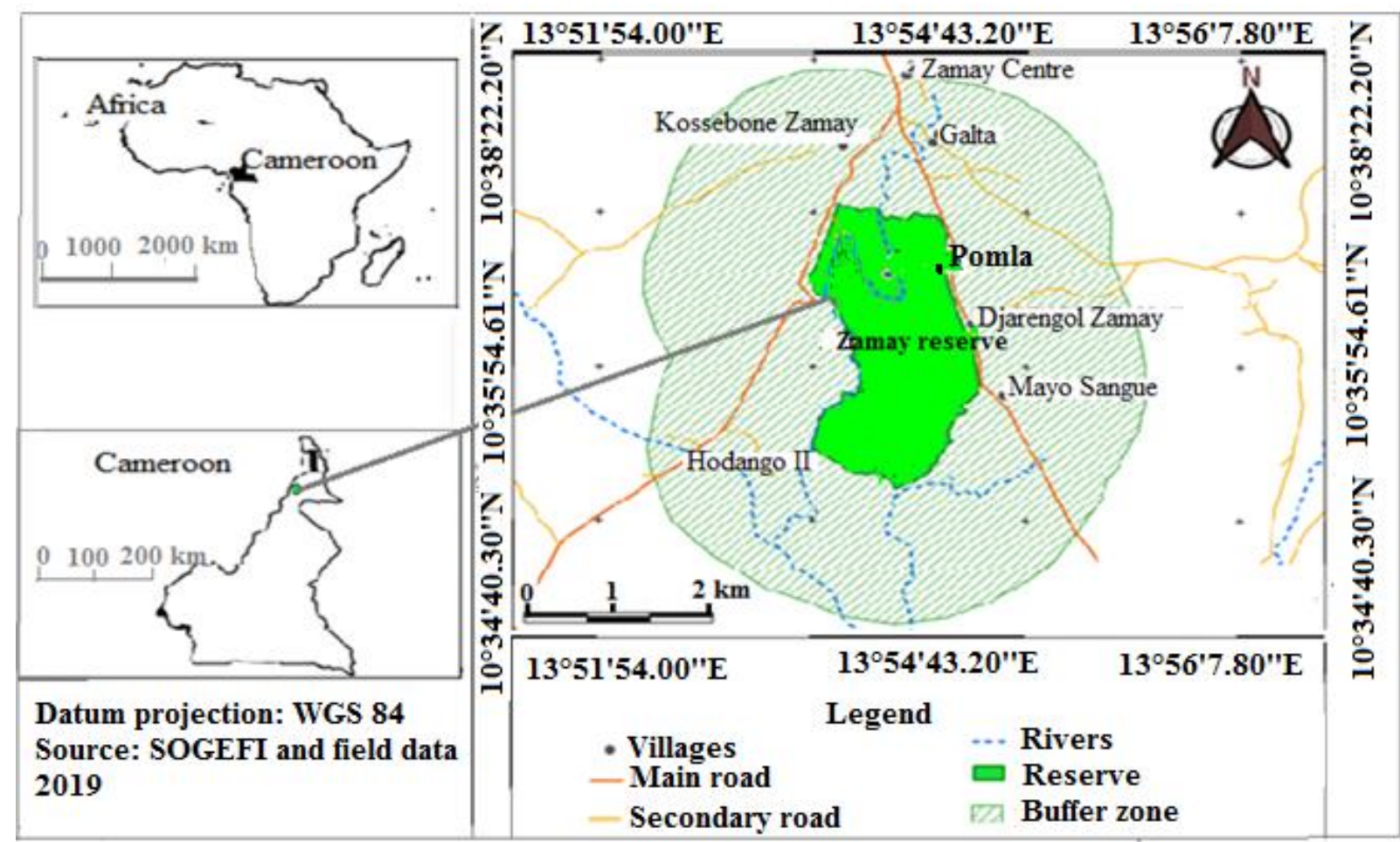

Figure 1: Map of location of the study site.

\section{Data collection}

\section{Inventory of tree plant resources in the reserve}

Study design

The inventory design is based on a method of biodiversity survey, in particular that of the plots developed by Picard et al. (2010). Three collection units (UC) constituting the treatments have been established in the reserve: $\mathrm{UC}_{1}$ in the northern part of the reserve, $\mathrm{UC}_{2}$ in the center and $\mathrm{UC}_{3}$ in the south of the reserve. In each collection unit, seven plots of $2500 \mathrm{~m}^{2}$ each $(50 \mathrm{~m} \times 50 \mathrm{~m})$ constituting the replications were established, making a total area of 5.25 ha. The space between two plots was $100 \mathrm{~m}$. In each plot, all the woody plants with a diameter at breast height $(\mathrm{dbh}) \geq 10 \mathrm{~cm}$ at $1.30 \mathrm{~m}$ were inventoried and identified by their scientific and local names. For pruning species, the average circumference of the stems was used to determine the diameter and the individuals that plug in before $1.30 \mathrm{~m}$ in height were measured at $10 \mathrm{~cm}$ from the ground (Jiagho et al., 2016).The height of species was estimated using a graduated pole and the circumference measured using a measuring tape. The survey was conducted in July 
2019. Identification of the species was done with the help of "Flora of West tropical Africa" (Tailfer, 1990), and the different volumes of "Flora of Cameroon" (Letouzey, 1985).

\section{Determination of local uses of plant non-timber forest products}

The local uses of NTFPs were determined on the basis of an ethnobotanical survey which took place in four localities around the reserve: Pomla II (24 respondents), Sabongari (24 respondents), Zamay center (30 respondents) and Kossehone (42 respondents). The selection of villages was made on the basis of their position around the reserve (North, South, East and West). A total of 120 available people aged of 15 and above, representing $30 \%$ of the local people, was interviewed. The main ethnic groups in the study area were Peuhl, Mafa and Moufou. The most dominant language is Fufuldé used by the Peuhl. We used participatory appraisal which consists of semi-structured and structured interviews developed by Blanchet and Gotman (2010). The following questions (Annex 1) were asked for the different NTFPs: the type of use, the materials for collection, the availability of the products in the Zamay forest reserve, the most important plants and finally the opportunities for sustainable management of resource species.

\section{Assessment of vulnerability status of tree species of non-timber forest products}

The vulnerability status was assessed through the 120 actors' perceptions involved in the collection of NTFPs. The following questions were asked: collected organs, domains of the use, mode of collection, stage of development of the organ, the interviewee's point of view on the state of abundance of the species, the possible causes of the scarcity of plant resources and proposals for strategies for the conservation of useful species. The three-level vulnerability scale, from 1 to 3, proposed by Betti (2001), was used to calculate species vulnerability: 1 for species with weak vulnerability; 2 for species with moderate vulnerability; 3 for a high vulnerable species.

\section{Data processing and analysis}

We assessed diversity of tree resource species with Shannon-Weaver diversity index (H') (Magurran, 2004) and Shannon's Evenness index (EQ). Diversity index takes into account not only the number of species but also whether species are more or less equally abundant, or whether in contrast one or a few species dominate.

$\mathrm{H}^{\prime}=-\Sigma \mathrm{Ni} / \mathrm{N} \log _{2} \mathrm{Ni} / \mathrm{N}$, where $\mathrm{H}^{\prime}=$ index of species diversity, $\mathrm{Ni}=$ number of individuals of a given species i, $\mathrm{N}=$ total number of individuals, $\log _{2}=\operatorname{logarithm}$ in basis 2 .

$\mathrm{EQ}=\mathrm{H}^{\prime} / \log _{2} \mathrm{~N}$, this index varies from 0 to 1 .

To describe the ecological importance of species within each collection unit as well as for the total flora, the species importance value index (IVI) (Mori et al., 1983), was also calculated:

Relative density $=($ Number of individuals of a species $(\mathrm{Ni}) /$ total number of individuals of all species $(\mathrm{N})) \times 100$

Relative frequency $=($ Frequency of a species/sum of all frequencies $) \times 100$

$\mathrm{IVI}=$ relative density + relative frequency + relative dominance

Relative dominance $=($ basal area of a species/basal area of all the species $) \times 100$

Basal area $(\mathrm{BA})=\sum_{\mathrm{i}}^{S}\left(\frac{\pi D^{2}}{4}\right)$.

Where D: diameter at breast height $(\mathrm{cm}) ; \pi: 3.141593$; BA: basal area.

For sarmenting species, the average circumference was calculated by the following formula:

$\mathrm{Cm}=\sqrt{\sum_{t=1}^{n}\left(\mathrm{Ci}^{2}\right)}$ (Todou et al., 2017).

Where $\mathrm{Cm}$ is the average circumference of stems; $\mathrm{Ci}$ is the circumference of a stem. 
The frequency of citation was calculated by the following formula:

$\mathrm{FC}=\frac{\mathrm{n}}{\mathrm{N}} \times 100$ (Gbekley et al., 2015).

Where FC is the frequency of citation; $n$ is the number of times the species was cited in the receipts; $\mathrm{N}$ is the number of interviewed persons.

The use value makes it possible to significantly determine the species with a high use value in a given environment. The use value of a species $\mathrm{i}$ within a use category $\mathrm{k}$ is represented by its average use score within this category. The use value of NTFPs was calculated by the following formula:

$$
\mathrm{Vu}(\mathrm{i})=\sum_{k=0}^{n}\left(\frac{\mathrm{Sk}}{\mathrm{n}}\right) \text { Camou }- \text { Guerrero et al (2008). }
$$

Where $\mathrm{Vu}$ is the use value, $\mathrm{Sk}$ is the number of positive responses for the use category $\mathrm{k}$ and $\mathrm{n}$ is the total number of positive responses for this use category of all the species studied.

The total use value of the species $i$ is then calculated by the sum of the use value of this species within the different use categories.

$\mathrm{VuT}=\sum_{\mathrm{i}=1}^{\mathrm{n}}(\mathrm{Vu}(\mathrm{i})$

Where VuT is the total use value of all the species and Vu the use value of a species.

The vulnerability index of the species (VIi) was calculated from the following formula (Betti, 2001):

VIi $=\mathrm{N} / 6$ with $\mathrm{N}=\mathrm{N}_{1}+\mathrm{N}_{2}+\mathrm{N}_{3}+\mathrm{N}_{4}+\mathrm{N}_{5}+\mathrm{N}_{6} ; \mathrm{N}_{1}=$ frequency of use; $\mathrm{N}_{2}=$ Number of uses; $\mathrm{N}_{3}=$ organ of plant used; $\mathrm{N}_{4}=$ method of collection; $\mathrm{N} 5=$ stage of development; $\mathrm{N}_{6}=$ relative frequency.

VIi $<2$, the species is weakly vulnerable; $2 \leq$ VIi $<2.5$, the species is moderately vulnerable; VIi $\geq 2.5$, the species is highly vulnerable (Table 1 ).

Table 1: Important parameters for vulnerability index (VI). Fm = maximal relative frequency (Adapted from Betti (2001)).

\begin{tabular}{|c|c|c|c|}
\hline \multirow[b]{2}{*}{ Parameters } & \multicolumn{3}{|c|}{ Vulnerability to an uncontrolled exploitation } \\
\hline & weak $($ scale $=1)$ & Moderate (scale $=2)$ & High $($ scale $=3)$ \\
\hline Frequency of use (FU) & $\mathrm{FU} \leq 20 \%$ & $20 \% \leq \mathrm{FU} \leq 60 \%$ & $\mathrm{FU} \geq 60 \%$ \\
\hline Number of uses (NU) & $\mathrm{NU} \leq 2$ & $2<\mathrm{NU}<4$ & $\mathrm{NU}>5$ \\
\hline $\begin{array}{l}\text { Organ of plant used } \\
\text { (OPU) }\end{array}$ & Leave, latex & Fruit, branch & $\begin{array}{l}\text { Wood, seed, bark, root, } \\
\text { flower }\end{array}$ \\
\hline Method of collection (MC) & Picking & - & Picking, logging \\
\hline Stage of development (SD) & Old or senescent & Adult & Young \\
\hline Relative frequency $(\mathrm{Fr})$ & $\mathrm{Fr} \geq 2 / 3 \mathrm{Fm}$ & $1 / 3 \mathrm{Fm} \leq \mathrm{Fr} \leq 2 / 3$ & $\mathrm{Fr} \leq 1 / 3 \mathrm{Fm}$ \\
\hline
\end{tabular}


Analysis of variance (ANOVA) was used to compare means of frequency of citation and use values of non-timber forest products. It was also used to compare the mean diversity measures between the collection units.

\section{Results}

\section{Diversity of tree resource species in Zamay forest reserve}

A total number of $745 \mathrm{stems}$ of $\mathrm{dbh} \geq 10 \mathrm{~cm}$ was recorded within the three collection units (CU) (Table 2), representing fifty-two species grouped in twenty-two families and thirtyseven (37) genera. The number of species per collection unit varied from twenty-five to fourtyfour species with a mean of $32.66 \pm 8.17$ species per collection unit. The number of species was lower in $\mathrm{CU}_{3}(\mathrm{n}=25)$ in the southern part of the reserve, moderate in $\mathrm{CU}_{2}$ in the center $(\mathrm{n}=29)$ and higher in $\mathrm{CU}_{1}$ in the north $(\mathrm{n}=44)$. The Shannon diversity index $\left(\mathrm{H}^{\prime}\right)$ values varied from 2.52 to 3.11, and Shannon evenness index (EQ) from 0.75 to 0.83 . For the whole collection units, they were 3.01 bits and 0.80 respectively. Shannon diversity index was moderate in $\mathrm{CU}_{1}$ $\left(\mathrm{H}^{\prime}=3.11\right)$ and weak in $\mathrm{CU}_{2}$ and $\mathrm{CU}_{3}\left(\mathrm{H}^{\prime}=2.52\right.$ and 2.68 respectively). Mean diversity measures differed significantly between the collection units (ANOVA, df $=6 ; p=0.004$ ).

Table 2: Structural parameters of the various collection units. H'= Shannon diversity index; $\mathrm{EQ}=$ Shannon evenness index $\mathrm{CU}$ : collection unit

\begin{tabular}{lllllll}
\hline Parameters & Species & Family & Genus & Number of stems & H' $^{\prime}$ & EQ \\
\hline $\mathrm{CU}_{1}$ & 44 & 20 & 32 & 314 & 3.11 & 0.82 \\
$\mathrm{CU}_{2}$ & 29 & 16 & 22 & 262 & 2.52 & 0.75 \\
$\mathrm{CU}_{3}$ & 25 & 14 & 20 & 169 & 2.68 & 0.83 \\
\hline Total & 52 & 22 & 37 & 745 & 3.01 & 0.80 \\
\hline
\end{tabular}

\section{Floristic composition of Zamay forest reserve}

The importance value index (IVI) analysis showed that Anogeissus leiocarpus (DC.) Guill. \& Perr., Boswellia dalzielii Hutch., Balanites aegyptiaca L. and Acacia hockii De Wild. were the most dominant species (Table 3). They represented $51.17 \%$ of the total IVI. The majority of the species (92.31\%) had IVI values less than eighteen, and sixteen had IVI less than one such as Gardenia aqualla Stapf \& Hutch., Acacia nilotica (L.) Willd. ex Del., A. polyacantha Willd., A. sieberiana DC., A. seyal Del., Pterocarpus lucens Guill. \& Perr., Steganotaenia araliacea Hochst., Bauhinia rufescens Lam., Combretum molle R. Br. ex G. Don, C. fragrans Steud. ex A Rich, Stereospermum kunthianum Cham., Piliostigma reticulatum (DC.) Hochst., Ziziphus mucronata Willd., Annona senegalensis Pers., Andira inermis (Wright) DC. and Grewia bicolor Juss.. The four families that had dominating species were Combretaceae (05 species); Burseraceae (02 species); Balanitaceae (01 species) and Mimosaceae (09). The average IVI in the different collection units were moderate in $\mathrm{CU}_{2}$ and $\mathrm{CU}_{3}$ (IVI $=10.32 ; 11.99$ respectively) but weak in $\mathrm{CU}_{1}$ (IVI= 6.94). Most of the dominating species were found in $\mathrm{CU}_{2}$ and $\mathrm{CU}_{3}$. The means of IVI in the different blocks were significantly different $(\mathrm{P}<0.05)$. 
Table 3: Importance value index (IVI) of tree species in each collection unit, and global IVI for all collection units in Zamay forest reserve. Bold in scientific name and family indicates the most important NTFPs and families in terms of IVI.

\begin{tabular}{|c|c|c|c|c|c|}
\hline \multirow[t]{2}{*}{ Species } & \multirow[t]{2}{*}{ Family } & \multirow[t]{2}{*}{ Global IVI } & \multicolumn{3}{|c|}{ IVI } \\
\hline & & & $\mathrm{CU}_{1}$ & $\mathrm{CU}_{2}$ & $\mathrm{CU}_{3}$ \\
\hline Acacia ataxacantha DC. & & 17.54 & 0.75 & 17.91 & 44.62 \\
\hline Acacia gerrardii Benth. & & 3.37 & 2.87 & 1.67 & 8.18 \\
\hline Acacia hockiiDe Wild. & & 29.29 & 3.62 & 4.71 & 25.38 \\
\hline Acacia nilotica (L.) Willd. ex Del. & & 0.30 & 21.74 & & \\
\hline Acacia polyacantha Willd. & Mimosaceae & 0.31 & 0.75 & & \\
\hline Acacia Senegal (L.) Willd. & & 4.85 & 2.8 & 35.96 & 1.20 \\
\hline Acacia seyal Del. & & 0.90 & 9.11 & 0.32 & \\
\hline Acacia sieberiana DC. & & 0.55 & 1.32 & & \\
\hline Dichrostachys cinerea (L.) Wright \& Arn. & & 0.12 & 2.04 & & 1.22 \\
\hline Andira inermis (Wright) DC. & & 0.85 & & & 3.79 \\
\hline Ptercarpus lucens Guill. \& Perr. & Fabaceae & 0.31 & 0.75 & & \\
\hline Annona senegalensis Pers. & Annonaceae & 0.59 & 1.42 & & \\
\hline Azadirachta indica A. Juss. & Meliaceae & 5.49 & 13.95 & & \\
\hline Khaya senegalensis(Desr.) A. Juss. & & 1.93 & 5.15 & & \\
\hline Balanites aegyptiaca (L.) Del. & Balanitaceae & 32.89 & 32.49 & 38.87 & 24.32 \\
\hline Bauhinia rufescens Lam. & & 0.34 & 0.83 & & \\
\hline Dalbergia melanoxylon Guill. \& Perr. & & 1.52 & 0.72 & 0.77 & 4.19 \\
\hline Dalbergia sissoo Roxb. & & 2.61 & 6.70 & & \\
\hline Senna singueana (Del.) Lock & Caesalpiniaceae & 1.98 & 2.65 & 2.45 & \\
\hline Senna siamea (Lam.) Irwin \& Barneby & & 1.07 & 2.65 & & \\
\hline Piliostigma reticulatum (DC.) Hochst. & & 0.39 & 0.98 & & \\
\hline $\begin{array}{l}\text { Piliostigma thonningii (Schumach.) Milne- } \\
\text { Redh. }\end{array}$ & & 1.48 & 3.68 & & \\
\hline Tamarindus indica $\mathrm{L}$. & & 16.02 & 21.34 & 11.49 & 16.37 \\
\hline Bombax costatum Pellegr. \& Vuillet & Bombacaceae & 2.69 & & 2.75 & 7.37 \\
\hline Boswellia dalzielii Hutch. & Burseraceae & 34.98 & 33.75 & 45.83 & 22.18 \\
\hline
\end{tabular}


Commiphora africana (A. Rich.) Engl. 4.06

Bridelia scleroneura Müll. Arg.

Euphorbia sudanica A. Chev.

1.13

Grewia bicolor Juss.

8.34

7.17

3.48

Flueggea virosa (Roxb. Ex Willd) Voigt

Cadaba glandulosa Forssk.

Capparis sepiaria L.

Anogeissus leiocarpus(DC.) Guill. \& Perr.

Combretum aculeatum Vent.

Combretum fragrans F. Hoffm.

Combretum glutinosum Perr. \& DC.

Combretum molle R. Br. ex G. Don.

Terminalia laxiflora Engl.

Diospyros mespiliformis Hochst. ex A. Rich.

Feretia apodanthera Del.

Gardenia aqualla Stapf. \& Hutch.

Ficus platyphylla Del.

Lannea acida A. Rich.

Lannea fruticosa (Hochst. ex A. Rich.) Engl.

Lannea humilis (Oliv.) Engl.

Sclerocarya birrea (A. Rich.) Hochst.

Steganotaenia araliacea Hochst.

Stereospermum kunthianum Cham.

Strychnos spinosa Lam.

Ximenia Americana L

Ziziphus mauritiana Lam.

Ziziphus mucronata Willd.

Euphorbiaceae

0.88

1.73

Capparaceae

2.19

8.33

56.36

2.49

0.39

Combretaceae

1.37

1.59

17.39

2.12

5.58

0.75

8.80

$\mathbf{3 8 . 5 6}$

0.67

2.64

2.49

0.79

7.67

$\mathbf{7 8 . 0 8}$

9.83

$\mathbf{5 3 . 4 9}$

9.75

1.74

$2.93 \quad 0.78$

$\begin{array}{ll}0.35 & 0.98\end{array}$

1.22

2.28

0.98

$\begin{array}{lllll}\text { Ebenaceae } & 3.32 & 2.28 & 3.82 & 1.24 \\ \text { Rubiaceae } & 8.11 & 3.18 & 7.01 & 16.18 \\ & 0.27 & 4.71 & 0.78 & \end{array}$

Moraceae

1.86

0.78

$\begin{array}{lll}4.17 & 8.69 & 1.62\end{array}$

$\begin{array}{lll}4.17 & 8.69 & 1.62 \\ 3.73 & & 1.92\end{array}$

3.73

Anacardiaceae $\quad 11.31$

18.13

4.69

8.76

6.34

$0.31 \quad 0.75$

$\begin{array}{lll}\text { Apiaceae } & 0.31 & 0.75 \\ \text { Bignoniaceae } & 0.37 & 0.92\end{array}$

Loganiacea

Olacaceae

Rhamnaceae

1.39

0.92

6.51

2.20

2.35

1.68

1.46
1.56

1.49

19.39

2.49 


\section{Local uses and use values of non-timber forest products}

Forty-four woody species of NTFPs were identified during the surveys and which were grouped into five classes based on their uses such as food $(31.75 \%)$, medicine $(50.79 \%)$, fodder $(12.70 \%)$, crafts $(3.17 \%)$ and fertilizers $(1.59 \%)$ (Figure 2$)$. Traditional medicine is at the forefront of healthcare for poor households. The majority of identified NTFPs resource plants was used for medicinal purposes $(50.79 \%)$. The parts of the plants such as bark, leaves, fruits, buds and roots were used as medicine. The oil extracted from the seeds of Khaya senegalensis (Desr.) A. Juss. and Azadirachta indica A. Juss. are used for several ailments such malaria, fever, anemia, rheumatism and diarrhea. The bark of Boswellia dalzielii Hutch. is used to treat wounds from snake bite. Decoction of leaves and roots of Detarium microcarpum Guill. \& Perr. is used for the treatment of rheumatism and malaria. The traditional medicine is followed by the category use of food which represents $31.75 \%$ of all the categories of uses. The different resource species are Haematostaphis barteri Hook. f., Tamarindus indica L., Sclerocarya birrea (A. Rich.) Hochst., Balanites aegyptiaca (L.) Del., Ziziphus mauritiana Lam., Ximenia americana L.and Diospyros mespiliformis Hochst ex A. Rich. They were mainly used for their fruits and are not only consumed for their organoleptic quality but also for their medicinal virtue. For example, fruits of Tamarindus indica L. used in the preparation of porridge are searched for treatment of stomachache.

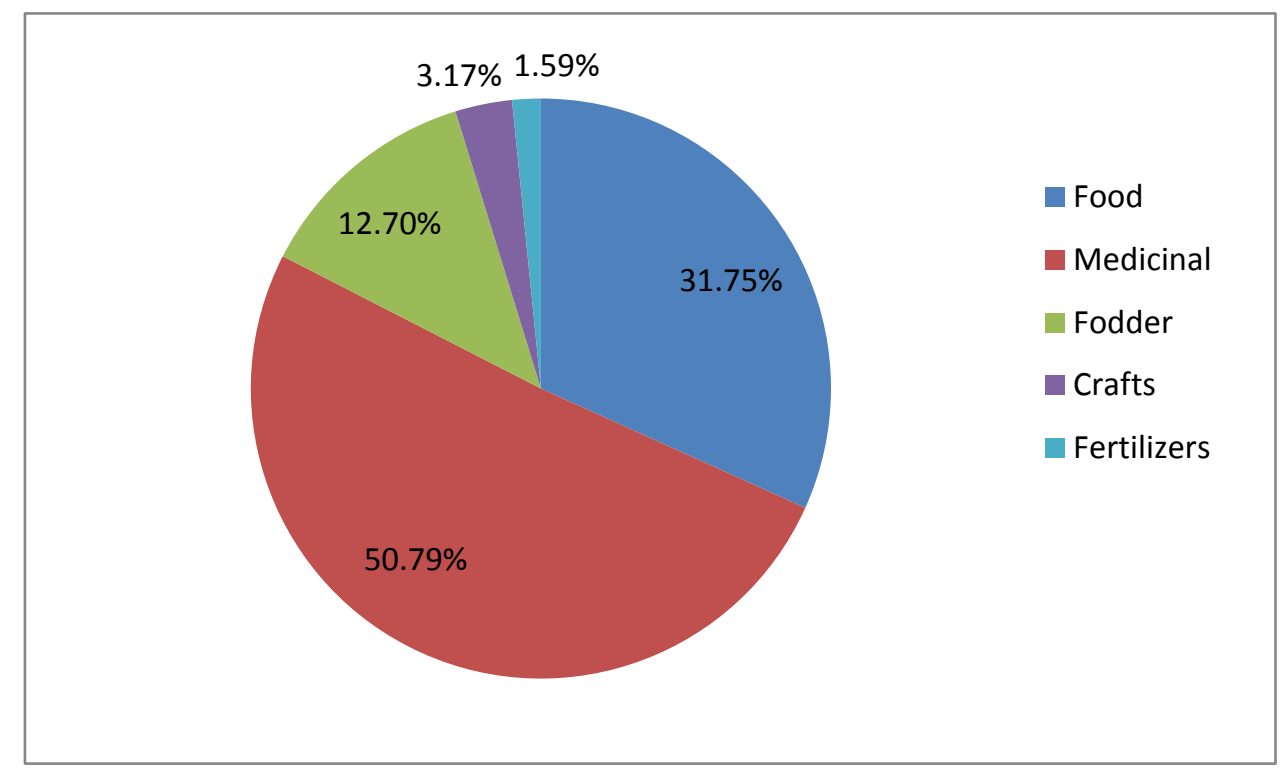

Figure 2: Rate of representation of categories of NTFPs uses.

The various NTFPs are either reproductive organs notably flowers, fruits and seeds, or vegetative organs constituted of stems, roots, barks, buds and leaves. As such, they were grouped into two categories namely reproductive organs and plant structures (Table 4). The most important used organs were fruits $(31.81 \%)$, leaves $(30.68 \%)$, barks $(22.73 \%)$ and the other parts such as stems, buds, roots, flowers, sap and seeds represented $14.8 \%$ of the frequency of citation made by respondents. 
Table 4: Various uses of non-timber forest products according to the collectors. The bold in scientific name indicates the most important NTFPs.

\begin{tabular}{|c|c|c|c|c|c|}
\hline \multirow{2}{*}{$\frac{\text { Fu: Fulfuldé }}{\text { Resource species }}$} & \multicolumn{5}{|l|}{ Mo: Moufou } \\
\hline & Local names & Collected organs & Methods of collection & Uses & $\begin{array}{l}\text { Frequency of citation } \\
(\%)\end{array}$ \\
\hline $\begin{array}{l}\text { Anogeissus leiocarpus (DC.) } \\
\text { Guill. \& Perr. }\end{array}$ & Kodjoli (Fu) & $\begin{array}{l}\text { Leaves, fruits, barks, } \\
\text { stems }\end{array}$ & debarking, & $\begin{array}{l}\text { Medicinal, fodder, } \\
\text { crafts }\end{array}$ & 52 \\
\hline $\begin{array}{l}\text { Senna siamea (Lam Irwin \& } \\
\text { Barneby }\end{array}$ & Forés $(\mathrm{Fu})$ & flowers & Picking & Medicinal & 34 \\
\hline $\begin{array}{l}\text { Khaya senegalensis(Desr.) A. } \\
\text { Juss. }\end{array}$ & Daleehi (Fu) & $\begin{array}{l}\text { Leaves, fruits, barks, } \\
\text { roots }\end{array}$ & Picking, debarking & Medicinal, fodder & 30 \\
\hline Haematostaphis barteri Hook f. & Toursoudje (Fu) & Fruits, barks, leaves & Picking, debarking & Medicinal, food & 27 \\
\hline Tamarindus indica $\mathbf{L}$. & Jabbe $(\mathrm{Fu})$ & Leaves, fruits, barks & Picking, debarking & Medicinal, food & 26 \\
\hline Azadirachta indica A. Juss. & $\begin{array}{l}\text { Neem (Fu, Ma, } \\
\mathrm{Mo})\end{array}$ & Leaves, fruits, stems & Picking, logging & medicinal & 23 \\
\hline Acacia albida Del. & Chaskii (Fu) & Leaves, fruits & picking & Fodder, fertilizer & 22 \\
\hline $\begin{array}{l}\text { Sclerocarya birrea (A. Rich.) } \\
\text { Hochst. }\end{array}$ & Eedi $(\mathrm{Fu})$ & $\begin{array}{l}\text { Leaves, fruits, barks, } \\
\text { seeds, stems }\end{array}$ & debarking, & Food, medicinal & 22 \\
\hline Balanites aegyptiaca (L.) Del. & Tanni $(\mathrm{Fu})$ & Leaves, fruits, seeds & Picking & Food, crafts & 20 \\
\hline Ziziphus mauritiana Lam. & Jaabi $(\mathrm{Fu})$ & Fruits, leaves, barks & Picking, debarking & $\begin{array}{l}\text { Medicinal, food, } \\
\text { fodder }\end{array}$ & 19 \\
\hline $\begin{array}{l}\text { Diospyros mespiliformis } \\
\text { Hochst. ex A. Rich. }\end{array}$ & Nelbi $(\mathrm{Fu})$ & Leaves, fruits, barks & Picking, debarking & $\begin{array}{l}\text { Medicinal, food, } \\
\text { fodder }\end{array}$ & 17 \\
\hline Vitex doniana Sweet. & Galbidje (Fu) & Fruits, barks & Picking, debarking & Food, medicinal & 17 \\
\hline Boswellia dalzielii Hutch. & Andakehi (Fu) & Barks & Debarking & Medicinal & 14 \\
\hline Ximenia americana $\mathrm{L}$. & Chabbuli (Fu) & Fruits & picking & Food & 7 \\
\hline $\begin{array}{l}\text { Detarium microcarpum Guill. \& } \\
\text { Perr. }\end{array}$ & Konkehi (Fu) & Leaves, fruits, barks & Picking, debarking & Food, medicinal & 6 \\
\hline Ficus polita Vahl. & Liitahi (Fu) & Leaves, fruits, barks & Picking, debarking & Food, medicinal & 6 \\
\hline Calotropis procera(Ait.) Ait. f. & Babambi (Fu) & Leaves, flowers, roots & Picking, logging & Medicinal & 5 \\
\hline $\begin{array}{l}\text { Eucalyptus camaldulensisF. } \\
\text { Muel. }\end{array}$ & $\begin{array}{l}\text { Kaliptus } \\
\text { Ma, Mo })\end{array}$ & Leaves & picking & Medicinal & 4 \\
\hline
\end{tabular}




\begin{tabular}{|c|c|c|c|c|}
\hline Anacardium occidentale L. & $\begin{array}{l}\text { Anakardiom } \\
(\mathrm{Fu}, \mathrm{Ma}, \mathrm{Mo})\end{array}$ & fruits & picking & Food \\
\hline $\begin{array}{l}\text { Piliostigma reticulatum (DC.) } \\
\text { Hochst. }\end{array}$ & Barkeehi (Fu) & Leaves, buds, roots & Picking, logging & Medicinal \\
\hline Jatropha curcas L. & Colkolaje (Fu) & Fruits, sap & Picking, logging & Medicinal \\
\hline Jatropha gossypiifolia L. & Colkolaje (Fu) & Fruits, sap & Picking, logging & Medicinal \\
\hline Crateva adansonii DC. & Samanaeki (Fu) & Leaves, fruits, barks & Picking, debarking & Food, medicinal \\
\hline $\begin{array}{l}\text { Prosopis africana(Guill. \& } \quad \& \\
\text { Perr.) Taub. }\end{array}$ & Kohi (Fu) & Barks, stems & Debarking, logging & Medicinal \\
\hline Adansonia digitata $\mathrm{L}$. & Bokki (Fu) & Leaves, fruits & Picking & Food \\
\hline $\begin{array}{l}\text { Parkia biglobosa (Jacq.)R.Br. ex } \\
\text { G.Don }\end{array}$ & Nareehi $(\mathrm{Fu})$ & Leaves, fruits, barks & Picking, debarking & Food, medicinal \\
\hline Vitellaria paradoxa C.F. Gaertn & Kareehi (Fu) & $\begin{array}{l}\text { Fruits, roots, flowers, } \\
\text { barks }\end{array}$ & $\begin{array}{l}\text { Picking, } \\
\text { logging }\end{array}$ & Food, medicinal \\
\hline Terminalia laxiflora Engl. & Zadeehi (Fu) & Barks & Barking & Medicinal \\
\hline $\begin{array}{l}\text { Daniellia oliveri (Rolf) Hutch. \& } \\
\text { Dalz. }\end{array}$ & Kayarlahi (Fu) & Barks, stems & Debarking, logging & medicinal \\
\hline $\begin{array}{l}\text { Ipomoea asarifolia (Desr.) } \\
\text { Roem. \& Schult. }\end{array}$ & $\begin{array}{l}\text { Daandi mayo } \\
(\mathrm{Fu})\end{array}$ & Leaves & Picking & Medicinal \\
\hline $\begin{array}{l}\text { Commiphora africana (A. Rich.) } \\
\text { Engl. }\end{array}$ & Badadi (Fu) & Barks & Debarking & Medicinal \\
\hline Borassus aethiopum Mart. & Dubbi (Fu) & Fruits, leaves & picking & Food, arts \\
\hline Annona senegalensis Pers. & Dukuhi (Fu) & Leaves, fruits & picking & Food, medicinal \\
\hline $\begin{array}{l}\text { Combretum molleR. Br. ex G. } \\
\text { Don }\end{array}$ & Sereehi $(\mathrm{Fu})$ & Roots, barks & Debarking, logging & Medicinal \\
\hline $\begin{array}{l}\text { Ficus sycomorus(Miq.) C.C. } \\
\text { Berg }\end{array}$ & Ibbe $(\mathrm{Fu})$ & Leaves, fruits & Picking & Medicinal \\
\hline Senna singueana (Del.) Lock & Yagueho $(\mathrm{Fu})$ & Leaves & Picking & medicinal \\
\hline Ziziphus mucronata Willd. & Gulum jabé (Fu) & Leaves, stems & picking & Food \\
\hline $\begin{array}{l}\text { Terminalia macropteraGuill. \& } \\
\text { Perr. }\end{array}$ & $\begin{array}{l}\text { Forafonguino } \\
(\mathrm{Fu})\end{array}$ & Barks & Debarking & Medicinal \\
\hline Ziziphus spina-christi (L.) Desf. & Kurnadje (Fu) & fruits & Picking & Food \\
\hline
\end{tabular}




\begin{tabular}{|c|c|c|c|c|}
\hline $\begin{array}{l}\text { Stereopermum kunthianum } \\
\text { Cham. }\end{array}$ & Golombi (Fu) & Leaves & Picking & Fodder \\
\hline Acacia sieberiana DC. & Aluki (Fu) & Leaves & Picking & Fodder \\
\hline Cissus quadrangularis L. & Gaadal $(\mathrm{Fu})$ & Fruits & Picking & Medicinal \\
\hline $\begin{array}{l}\text { Hexalobus monopetalus (A. } \\
\text { Rich.) Engl. \& Diels }\end{array}$ & Bohili (Fu) & Fruits & Picking & Food \\
\hline $\begin{array}{l}\text { Acacia nilotica (L.) Willd. ex } \\
\text { Del. }\end{array}$ & $\begin{array}{l}\text { Gabde } \\
(\mathrm{Fu})\end{array}$ & Leaves, fruits & Picking & Medicinal, food \\
\hline
\end{tabular}


The consumed parts of the plants for medicinal purpose were fruits, barks, roots, stems, flowers, leaves, buds and sap. The most important used parts were leaves (27.94\%), barks (27.94\%) and fruits $(23.52 \%)$ (Figure 3). These three parts of the plants represented $79.40 \%$ and the other parts of the plants such as roots, buds, stems, flowers and sap recorded $20.60 \%$ on the whole.

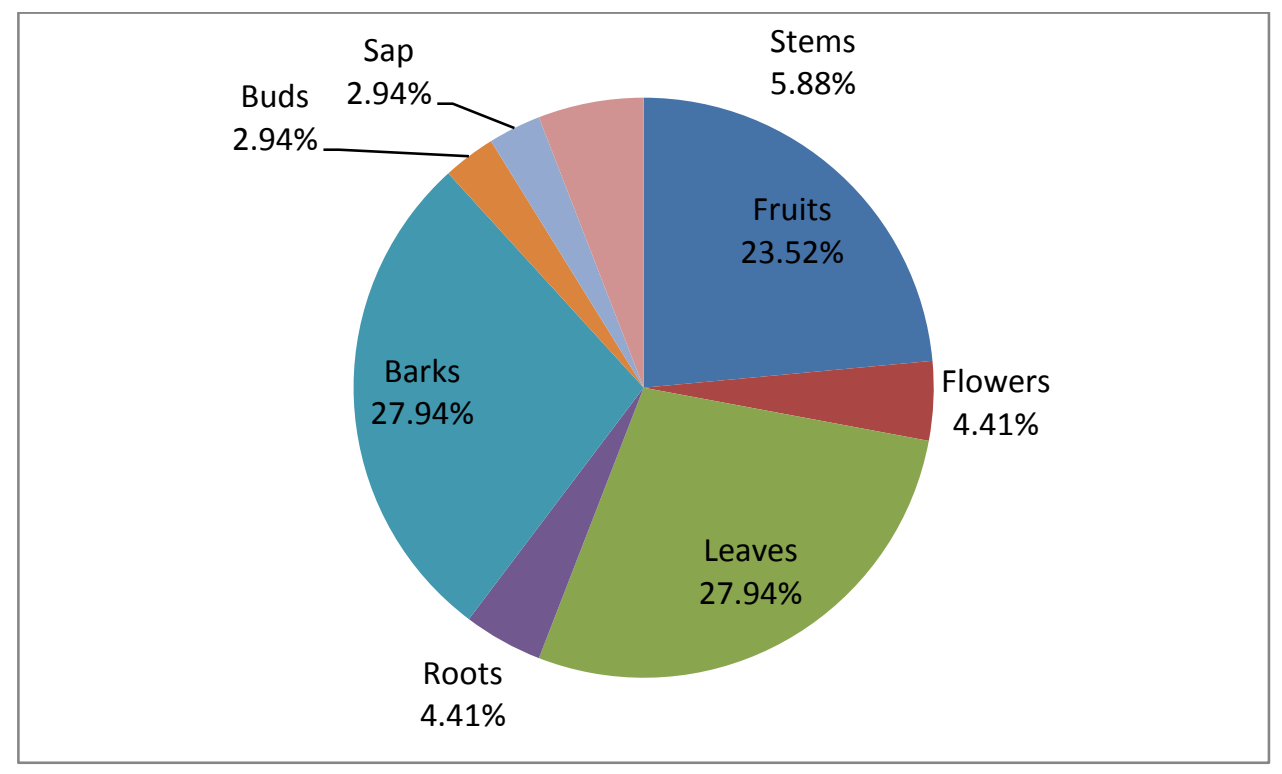

Figure 3: Rate of the consumed parts of plants for medicinal use.

The seven most important species (Table 5) in terms of total use values were: Acacia albida Del. (VuT = 1.42); Anogeissus leiocarpus (DC.) Guill. \& Perr. (VuT = 0.96); Khaya senegalensis (Desr) A. Juss. (VuT =0.33); Balanites aegyptiaca (L.) Del. (VuT =0.33); Ziziphus mauritiana Lam. (VuT =0.23); Tamarindus indica L. $(\mathrm{VuT}=0.21)$ and Sclerocarya birrea (A. Rich.) Hochst. (VuT = 0.21). These tree species represented $69.85 \%$ of the total use values and constituted the NTFPs with high use value of the study site. About fifteen species such as Acacia nilotica (L.) Willd. ex Del., Senna singueana (Del.) Lock, Cissus quadrangularis L., Combretum molle R. Br. ex G. Don, Commiphora africana (A. Rich.) Engl., Daniellia oliveri (Rolfe) Hutch. \& Dalz., Ficus sycomorus (Miq.) C.C. Berg, Detarium microcarpum Guill. \& Perr., Hexalobus monopetalus (A. Rich.) Engl. \& Diels, Ipomoea asarifolia (Desr.) Roem. \& Schult., Jatropha gossypiifolia L., Terminalia laxiflora Engl., T. macroptera Guill. \& Perr., Ziziphus mucronata Willd. and Z. spina-christi (L.) Desf. were recorded as a very less use value and represented $1.58 \%$ of the total use value. The less used NTFPs were all characterized by only one category of uses. Means of frequency of citation (species are cited for different uses and receipts by respondents) and use values were not significantly different at 0.05 level (ANOVA, $\mathrm{df}=86 ; \mathrm{p}=0.30$ ). Rates of frequency of citation of local uses of products are positively correlated to total use values of plant resources (Pearson, $\mathrm{r}=0.84)$. 
Table 5: Use values of censused species. Bold in scientific name indicates the most important NTFPs. Vu $=$ use value, VuT $=$ total use value.

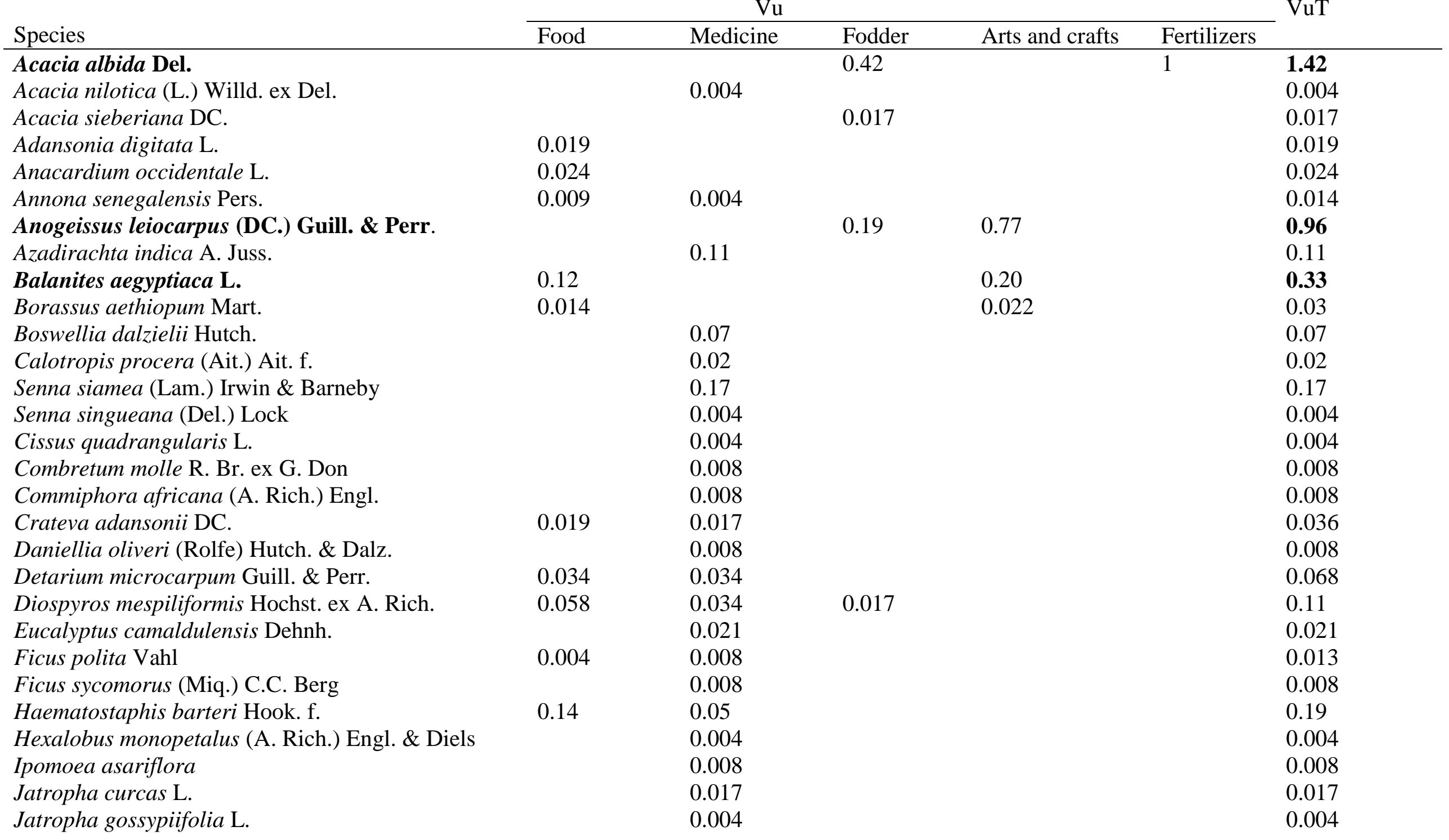


Khaya senegalensis (Desr.) A. Juss.

Parkia biglobosa (Jacq.) R. Br. ex G. Don

0.12

0.008

0.21

$\mathbf{0 . 3 3}$

Piliostigma reticulatum (DC.) Hochst.

0.004

0.021

0.013

Prosopis africana (Guill. \& Perr.) Taub.

0.017

0.021

Sclerocarya birrea (A. Rich.) Hochst.

0.05

0.05

0.017

Stereospermum kunthianum Cham.

0.11

0.017

0.21

\section{Tamarindus indica $\mathbf{L}$.}

0.18

0.03

Terminalia laxiflora Engl.

0.008

0.21

Terminalia macroptera H. Perr.

Vitellaria paradoxa Gaertn. f.

0.004

0.008

0.008

0.004

0.008

Vitex doniana Sweet

0.04

0.02

Ziziphus mauritiana Lam.

0.13

0.02

0.07

$\mathbf{0 . 2 3}$ 


\section{Vulnerability status of tree plant resources of non-timber forest products}

High use values of tree plant resources lead always to high pressure on the species. Eight species such as Acacia albida Del., Balanites aegyptiaca L., Diospyros mespiliformis Hochst. ex A. Rich., Ziziphus mauritiana Lam., Haematostaphis barteri Hook. f., Khaya senegalensis (Desr.) A. Juss., Senna siamea (Lam.) Irwin \& Barneby and Tamarindus indica L. were weakly vulnerable $(\mathrm{VI}<2)$. Two resource species such as Sclerocarya birrea (A. Rich.) Hochst and Anogeissus leiocarpus (DC.) Guill. \& Perr. were moderately vulnerable $(\mathrm{VI} \geq 2)$ (Table 6).

Table 6: Vulnerability status of the ten most important tree resource species. Bold in the scientific name indicates the most vulnerable NTFPs.

\begin{tabular}{lc}
\hline Species & Vulnerability index of the species (Vli) \\
\hline Acacia albida Del. & 1.40 \\
Anogeissus leiocarpus (DC.) Guill. \& Perr. & $\mathbf{2 . 4}$ \\
Balanites aegyptiaca L. & 1.4 \\
Diospyros mespiliformis Hochst ex A Rich. & 1.6 \\
Haematostaphis barteriHook. f. & 1.8 \\
Khaya senegalensis (Desr.) A. Juss. & 1.8 \\
Sclerocarya birrea (A. Rich.) Hochst. & $\mathbf{2 . 2}$ \\
Senna siamea (Lam.) Irwin \& Barneby & 1.8 \\
Tamarindus indica L. & 1.8 \\
Ziziphus mauritiana Lam. & 1.6 \\
\hline
\end{tabular}

\section{Discussion}

\section{Tree diversity of Zamay forest reserve}

Forest communities considered rich (Kent and Coker, 1992) are characterized by a Shannon diversity value $\left(\mathrm{H}^{\prime}\right)$ of about 3.5 or higher. The three collection units established within the Zamay forest reserve showed different values of Shannon's index (Table 2). In $\mathrm{CU}_{2}$ and $\mathrm{CU}_{3}$ respectively in the center and southern part of the reserve, Shannon diversity values were weak $\left(\mathrm{H}^{\prime}<3\right)$ because of the refugees and internal displaced persons who have an enormous impact on the vegetation of the south and center of the protected area. Shannon diversity value was moderate in $\mathrm{CU}_{1}$ situated at the northern part of the reserve because of the moderate anthropogenic activities at that level. Such value in savannah indicates a relative stability for the experimental year. The high evenness values observed in the site $(0.6 \leq \mathrm{EQ} \leq 0.8)$ indicates its level of maturity. The most important species in terms of IVI values were found in the families of Balanitaceae, Burseraceae, Combretaceae and Mimosaceae. The importance of these families in the study site is due to the fact that drought in the Sahel has allowed natural selection of the most robust species like in these families. Similarly, the surveys conducted in African savannahs and more typical in sudano-sahelian zones of BurkinaFaso and Cameroon (Bognounou et al., 2009; Froumsia et al., 2012) stated that these families are resistant to the lack and insufficient rains but also to high temperatures. Moreover, they are the most common and highly 
represented in tropical countries. Besides, the higher regeneration potential of those families is due to maximum seed dispersal capability (Jannat et al., 2020) and favorable conditions prevailing for natural regeneration.

\section{Importance of non-timber forest products in Zamay forest reserve}

The Zamay forest reserve area hosts valuable resource species of NTFPs used as food, fodders, medicines, crafts and arts and fertilizers. A total of fourty-four wood resource species exploited by the local populations was censused in the area. We found less number of species than that found by Guigma et al. (2012) who determined 147 useful species in the south of Burkina-Faso. The significant difference should be explained by the fact that the study of Guigma et al. (op. cit.) took into account the herbaceous vegetation. Similar study carried out in the agrosystems of sudano-sahelian zone of Cameroon (Kamblaba, 2018) obtained fourty-nine important ligneous species used by the local populations, which is similar to our results.

Two categories of product of NTFPs were identified: reproductive organs (flowers, fruits, seeds) and plant structures (stems, leaves). The products are used predominantly for traditional pharmacopoeia (50.79 $\%$ ) and food (31.75\%) (Figure 2). Two reasons such as a low level of purchasing power of consumers, and the expensive costs of pharmaceutical products explain the high solicitation of these two categories of uses. Forests significantly contribute to the nutrition and healthcare of local populations. The study on rural livelihood benefits from Participation in Taungya Agroforestry System (Adekunle and Bakare, 2009) argued that in Nigeria, majority of rural households and large proportion of urban households depend on forest products to meet parts of their nutritive and sanitary needs. Similarly, the survey on NTFPS at the periphery of Mbam and Djerem national park in Cameroon (Souare, 2015) mentioned that food and traditional medicine recorded both $62.67 \%$.

Seven resource species (Table 5) recorded the high use values and as such were considered as the most appreciated NTFPs in this study. The surveys on contribution of local tree species in sustaining the rural livelihoods in Burkina-Faso and Cameroon (Thiombiano et al., 2012; Mapongmetsem et al., 2012; Kamblaba, 2018) stated that these species are among the most appreciated ones in the sudano-sahelian and sudano-guinean zones of Cameroon, and in the sudano-sahelian zone of Burkina-Faso. Leaves, fruits and barks (Figure 3) were the most useful organs in our study site for their medicinal properties. Studies on plant system storage and phytochemistry (Georges et al., 2013; Mangambu et al., 2014; Sahli, 2017; Kouchadé et al., 2017) stated that organ collected on a species is related to the utility searched out by population and local knowledge of the species. Frequent use of leaves is justified by the abundance of chemical groups that they contain. Leaves are the sites of plant secondary metabolites synthesis. These results show the degree of dependence on NTFPs (Tsafack, 2016; Ketchatang et al., 2017) which vary according to the standard of living 
of the populations and their activities. This would also explain the gradient of differentiation of pressures from local populations on forest resources.

\section{Vulnerability status of tree plant resources}

Amongst the most important resource species of the area of Zamay forest reserve, two species were found vulnerable with a vulnerability index (VI) $\geq 2$ (Table 6), such as Anogeissus leiocarpus (DC.) Guill. \& Perr. and Sclerocarya birrea (A. Rich.) Hochst. because more than $90 \%$ of the species are systematically felled or barked. Eight species were found weakly vulnerable because the collection methods of the organs, mainly fruits, are done properly by picking up. For all of these NTFPs, extractivism is taking place both at the periphery and inside the reserve. Similarly, the surveys on uses and vulnerability of woody species in BurkinaFaso (Hahn-Hadjali and Thiombiano, 2000; Traoré et al., 2011) argued that Khaya senegalensis (Desr.) A. Juss. and Sterculia setigera Del. were vulnerable because of the collection of bark. Moreover, Prosopis africana (Guill. \& Perr.) Taub., Diospyros mespiliformis Hochst. ex A. Rich., Detarium microcarpum Guill. \& Perr. and Anogeissus leiocarpus (DC.) Guill. \& Perr. were vulnerable because of logging. In fact, intensive barking leads to loss of vigor of the plants which can scarcely assure their physiological functions at the level of their potentialities. Also, the increasing number of uses of species could make them vulnerable.

\section{Limitations of the study}

In the context of this study, we did not have the opportunity to interview an important group of people, i.e the refugees and the internally displaced persons fleeing the abuses of Boko-Haram, installed in Minawaou camp. This group of people has a great impact on the reserve resources. A future study will be addressed specifically to these people and the anthropogenic effects on their immediate environment will be raised.

\section{Conclusions}

Non-timber forest products have been widely recognized in sub-Saharan Africa as a source of significant livelihood value, especially for rural people, since they generate cash incomes, local medicines, supplementary food and other products required daily. A total number of 745 stems ( $\mathrm{dbh} \geq 10 \mathrm{~cm})$ of woody species was recorded within the three collection units installed in the reserve, representing fifty-two species grouped in twenty-two families and thirty-seven genera. The Shannon diversity index ( $\left.H^{\prime}\right)$ values varied from 2.52 to 3.11, and Shannon evenness index (EQ) from 0.75 to 0.83 . The four families which had the most dominating species were Combretaceae (05 species), Burseraceae (02 species), Balanitaceae (01 species) and Mimosaceae (09). The area of Zamay forest reserve hosted fourty-four useful resource species of non-timber forest products for the local populations, which were grouped into five categories of uses such as food, 
medicine, fodder, crafts and fertilizers. We found seven important resource species in terms of total use values and amongst them, two were vulnerable with VI $>2$. Rates of frequency of citation of useful products were positively correlated to total use values of plant resources. Results of this survey will allow considering reforestation with valued species such as Anogeissus leiocarpus (DC.) Guill. \& Perr. and Sclerocarya birrea (A. Rich.) Hochst. (Annex 2) in the degraded parts of the reserve. Efforts should be made more in order to improve the protection of the forest reserve, the development of a suitable regional conservation of the biodiversity and the domestication of the valued species.

\section{Acknowledgements}

The authors would like to recognize the support of International Foundation for Science (IFS) through a grant given to Dr. Souare Konsala $\left(\mathrm{N}^{\circ} \mathrm{D} / 4979-2\right)$. The materials provided by the Foundation permitted to perform this study. We are also grateful to the local communities for their participation.

\section{Conflicts of interest}

The authors declare that they have no conflict interest.

\section{Authors contribution statement}

Souare Konsala and Sassouang Evele Moctar carried out the study and developed the manuscript.The other authors, namely Froumsia Moksia, Hamawa Yougouda, Tchobsala and Ibrahima Adamou read and made comments that improved the manuscript.

\section{References}

Adekunle, V.A.J. and Bakare, Y., 2004. Rural Livelihood Benefits from Participation in Taungya Agroforestry System in Ondo State, Nigeria. Journal of Small-Scale Forest Eco-Management and Policy, 3(1): 131-138. DOI : 10.1007/s11842-004-0009-y.

Ayuk, E.T., Duguma, B., Franzel, S., Kengue, J., Mollet, M., Tiki-Manga, T. and Zenkeng, P., 1999. Uses, management and economic potential of Irvingia gabonensis in the humid lowlands of Cameroon. Forest Ecology and Management, 113(1) 1-9. DOI :10.1016/S0378-1127(98)00323-5

Bawa, K.S. and Gadgil, M., 1997. Ecosystem services, subsistence economies and conservation of biodiversity. In: Daily G, editor. Nature's services: societal dependence on natural systems. Washington (DC): Island Press. pp 295-310. 
Beer, J.H. and McDermott, M.J., 1989. The economic value of non-timber forest products in Southeast Asia. NC-IUCN, Amsterdam, the Netherlands. 175 p.

Belcher, B., Ruiz-Perez, M. and Achidiawan, R., 2005. Global patterns and trends in the use and management of commercial NTFPs: implications for livelihoods and conservation. World Development, 33(9) : 1435-1452. DOI:10.1016/j.worlddev.2004.10.007.

Betti, J.L., 2001. Vulnérabilité des plantes utilisées comme antipaludiques dans l'arrondissement de Mintom au sud de la réserve de Biosphère du Dja (Cameroun). Systematics and Geography of Plants, 71(2): 661-678. DOI: 10.2307/3668709.

Blanchet, A. and Gotman, A., 2010. L'entretien: L'enquête et ses méthodes. Paris : Armand colin. 128 p.

Bognounou, F., Thiombiano, A., Savadogo, P., Boussim, J.I., Odén, P.C., Guinko, S., 2009. Woody vegetation structure and composition at four sites along latitudinal gradient in Western Burkina Faso. Bois et Forêts des Tropiques, 300: 29-44. DOI : 10.19182/bft2009.300.a20412

Camou-Guerrero A., Reyes-Garcia., Martinez-Ramos, M.and Casas A., 2008. Knowledge and the value of plant species in a Raramuri community: a gender perpective for conservation. Human ecology, 36(2): 259-272. DOI : $10.1007 / \mathrm{s} 10745-007-9152-3$.

Djeukam, R., 2007. Le Cadre Legislatif et Reglementaire de L'utilisation des produits forestiers nonligneux au Cameroon. Renforcement de la sécurité alimentaire en Afrique Centrale à travers la gestion et l'utilisation durable des produits forestiers non-ligneux. Etude FAO. 47 p.

Dovie, D.B.K., Shackleton, C.M. and Witkowski, E.T.F., 2002. Direct-use values of woodland resources consumed and traded in a South African village. International Journal of Sustainable Development and World Ecology, 9(3): 269-283. DOI: https://doi.org/10.1080/13504500209470122.

Froumsia, M., Zapfack, L., Mapongmetsem, P.M. and Nkongmeneck, B.A.. 2012. Woody species, composition, structure and diversity of vegetation of Kalfou Forest reserve, Cameroon. Journal of Ecology and the Natural Environment, 4(13): 333-343. DOI: 10.5897/JENE12.047.

Gbekley, E.H., Karou, D.S., Gnoula, C., Agbodeka, K., Anani, K., Tchacondo, T., Agbonon, A., Batawila, K. and Simpou, J., 2015. Etude ethnobotanique des plantes utilisées dans le traitement du diabète dans la médecine traditionnelle de la région Maritime du Togo. Pan African Medicinal Journal, 20 : 437, pp 1-16. 
Georges, L., Gueye, M.T., Dogo, S. et Jean-Paul, W., 2013. Typologie des systèmes de stockage et de conservation du maïs dans l'Est et le Sud du Sénégal. Biotechnology, Agronomy, Society and Environment, 16(1): 49-58.

Guigma, Y., Zerbo, P. and Jeanne, M.R., 2012. Utilisation des espèces spontanées dans trois villages contigus du Sud du Burkina-Faso. Tropicultura 30: 230-235.

Hahn-Hadjali , K. and Thiombiano, A., 2000. Perception des espèces en voie de disparition en milieu Gourmantché (Est du Burkina Faso). Berichte des Sonderforschungsbereichs, 268, Band 14, Frankfurt. pp 285-297.

Hedge, R., Suryaprakash S., Achoth L. and Bawa K.S., 1996. Extraction of NTFPs in the forests of Biligiri Rangan Hills, India. Contribution to rural income. Economic Botany, 50(3): 243-251. DOI : 10.1007/BF02907328.

Jannat, M., Kamruzzaman, M. and Kamal Hossain, M., 2020. Assessment of natural regeneration potential of native tree species in a community management forest of Bangladesh. International Journal of Environment, 9(1): 100-114. DOI: https://doi.org/10.3126/ije.v9i1.27598.

Jiagho, E.R., Zapfack, L., Kabelong, B.L.P.R., Tsayem, D., Corbonnois, J. et Tchawa, P., 2016. Diversité de la flore ligneuse à la périphérique du Parc National de Waza (Cameroun). Revue des Sciences Environnementales, 6(1): 1-32. DOI: https://doi.org/10.4000/vertigo.17249.

Kamblaba, P., 2018. Diversité, structure et ethnobotanique quantitative des plantes ligneuses protégées et plantées dans les agrosystèmes saisonniers de l'Arrondissement de Méri (Extrême-Nord, Cameroun). Mémoire de Master 2 en Biologie Végétale, Université de Maroua. 53 p.

Kent, M. and Coker, P., 1992. Vegetation description and analysis. A practical approach, New York: John Wiley and Sons. 363 p.

Ketchatang, P.T., Zapfack, L., Kabalong, B.L.P. et Endama, D., 2017. Disponibilité des Produits Forestiers Non-Ligneux fondamentaux à la périphérie du Parc National de Lobeke. Vertigo, 17(3) : https://doi.org/10.4000/vertigo.18770

Kouchadé, S.A, Adjatin, A.R., Cossi-Adomou, A., Dassou, H.G. and Akoégninou, A., 2017. Phytochimiques des plantes médicinales utilisées dans la prise en charge des maladies infantiles au Sud-Bénin. European Scientific Journal, 13(3) : 471-488. DOI: 10.19044/esj.2016.v13n3p471 
Letouzey, R., 1985. Notice de la carte phytogéographique du Cameroun au 1: 500 000. Institut de la carte internationale de la végétation. Toulouse, France. pp. 63-142.

Magurran, A.E., 2004. Measuring biological diversity. Blackwell Publishing: Oxford, UK p. 256.

Mangambu, M.J., Mushagalusa, K.F., Kadima, N.J., 2014. Contribution à l'étude phytochimique de quelques plantes médicinales antidiabétiques de la ville de Bukavu et ses environs. Journal of Applied Biosciences, 75 : 6211-6220.

Mapongmetsem, P.M., Kapchie, V.N. and Tefemba, B.H., 2012. Diversity of local fruit trees and their contribution in sustaining the livelihood in the northern Cameroon. Ethiopian Journal of Environmental Studies and Management, 5(1): 32-46. DOI: http://dx.doi.org/10.4314/ejesm.v5i1.5.

Mori, S.A., Boom, B.M, Carvalino, A.M. and Dos Santos, T.S., 1983. The ecological importance of Myrtaceae in an eastern Brazilian wet forest. Biotropica, 15:68-70.

Ndangalasi, H.J., Bitariho, R. and Dovie, D.B.K, 2007. Harvesting of non-timber forest products and implications for conservation in two montane forests of East Africa. Bilogical Conservation, 134: 242-250. DOI:10.1016/j.biocon.2006.06.020.

Peters, C.M., 1990. Plenty of fruit but no free lunch. Garden, 14: 8-13.

Picard, J.F., Magnussen, S., Banack, N.L., Namkosserena, S. and Yalibanda, Y., 2010. Permanent sample plots for natural tropical forest: a rational with special emphasis on Central Africa. Environmental monitoring and assessment, 164 (1): 279-295. DOI: 10.1007/s10661-009-0892-y.

Rasul, G., Karki M. and Sah, R.P., 2008. The role of non-timber forest products in poverty reduction in India: Prospects and problems. Development Practices, 18(6): 779-88. DOI: https://doi.org/10.1080/09614520802386876.

Sahli, R., 2017. Etudes phytochimiques de quelques plantes extrémophiles Tunisiennes et exploration de leurs activités biologiques. Thèse de Doctorat, Université de Lille 2. 255 p.

Samantha, B. and Njikam, B.E., 2017. Impact environnemental du Camp des réfugiés de Minawaou. Rapport du Programme Alimentaire Mondial, URD. 49 p. 
Shackleton, C.M,, Guthrie G. and Main R., 2005. Estimating the potential role of commercial over-harvesting in resource viability: case study of five useful tree species in South Africa. Land Degradation and Development, 16(3): 273-286. DOI: DOI: https://doi.org/10.1002/ldr.652.

Souare, K. 2015. Gestion intégrée des espèces ressources clés des produits forestiers non-ligneux végétaux au Cameroun : cas de la périphérie du parc national du Mbam et Djerem. Thèse de Doctorat/PhD, Univ. Yaoundé I. 155 p.

Suchel, J.B. 1987. Rainfall patterns and regimes rainfall in Cameroon. Tropical Geography CEGET-CNRS, Talence. $287 \mathrm{p}$.

Tailfer, Y., 1990. La forêt dense d'Afrique Centrale: Approche botanique et systématique. Wageningen, CTA. $1271 \mathrm{p}$.

Téwéché, A., Ganota, B. et Gonné, B. 2016. Dynamique régressive de la végétation ligneuse dans une réserve forestière: cas de Zamay, Mayo-Tsanaga (Extrême-Nord, Cameroun). Commission des Forêts d'Afrique Centrale. pp 181-207.

Thiombiano, D.N.E., Lamien, N., Dibong, D.S., Boussim, I.J. and Belem, B., 2012. Le rôle des espèces ligneuses dans la gestion de la soudure alimentaire au Burkina-Faso. Sécheresse, 23(2): 86-93.

Ticktin, T., 2005. Applying a metapopulation framework to the management and conservation of a nontimber forest species. Forest Ecology and Management, 206(3): 249-261. DOI: https://doi.org/10.1016/j.foreco.2004.11.004.

Todou, G., Dedangsou, S. and Kemeuze, V.A,, 2017. Floristic composition, diversity and ecological importance of woody plants in eastern part of national park of Sena Oura, Chad. Journal of Biodiversity and Environmental Sciences, 11(2): 92-104.

Traoré, L., Ouédraogo, I., Ouédraogo, A. et Thiombiano, A., 2011. Perceptions, usages et vulnérabilité des ressources végétales ligneuses dans le Sud de Burkina-Faso. International Journal of Biological and Chemical Sciences, 5(1): 258-78. DOI: 10.4314/ijbcs.v5il.68103.

Tsafack, D.R., 2016. Integrated model of the sustainable contribution of Non-Timber Forest Products valorization on the livelihood assets of local people: The case of the northern periphery of the Dja reserve. Master Thesis, University of Yaoundé II. 111 p. 


\section{Annex 1}

\section{Questionnaire Sheet}

$\mathrm{N}^{\circ}$.

Date.

\section{I-Identification}

1.First Name and Name

2.Age

3.Sex:

4.Ethnic group :

5.Religion :

\section{II-Characteristics Of Used Plant Organs}

1.What are the different plant resources and the products that you use ? Complete the chart

\begin{tabular}{|l|l|l|}
\hline Local name & Scientific name & Collected parts \\
\hline a- & & \\
c- & & \\
d- & & \\
e- & & \\
f- & & \\
g- & & \\
$\ldots . . . .$. & & \\
\hline
\end{tabular}

2.What are the purposes of the uses of the products?
$\square$ Food
$\square$ Medicine
$\square$ Fodder
Rite
$\square$ Construction
$\square$ Others (to precise)

3. Are there any medicinal toxic plants?

4.At what period do you collect the parts of the plants?

— Rainy season

$\square$ Drought season

\section{Management of the resources}

5. How do you collect the products? Are there any specific materials?

Enumerate.

6. Where do you collect the products?
— Orchard
$\square$ Forest Reserve
Bush 
7. How do you evaluate the availability of the products in your area?

$\square$ Less $\square$ Moderate $\quad \square$ Sufficient

8.Do you know the limit of the Zamay Forest Reserve?

Please, describe.

9. Do you often collect products there?

10. What are the most important plant resources in your area? And why?

11.What do you propose for the sustainable management of the plant resources in your area?

12. Add other opportunities for sustainable management of the Zamay Forest Reserve.

Thank you for your contribution 
Annex 2

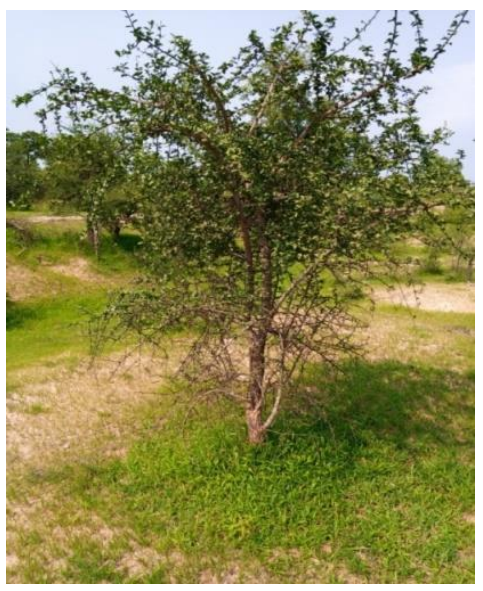

Balanites aegyptiaca L.

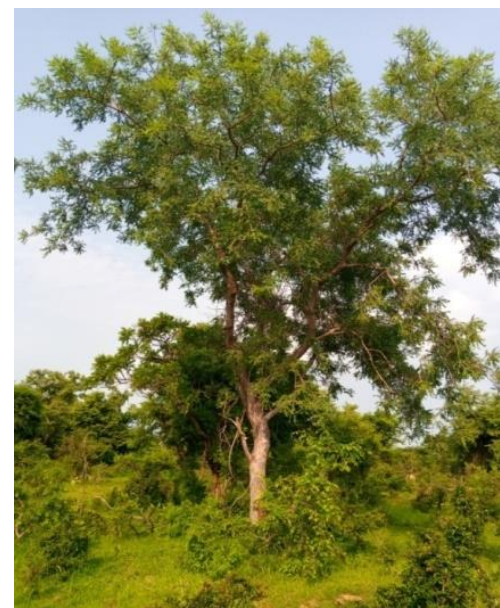

Sclerocarya birrea (A. Rich.) Hochst

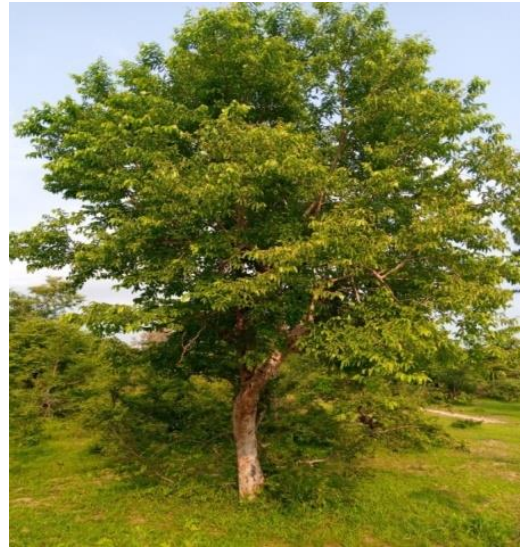

Anogeissus leiocarpus (DC.) Guill. \& Perr.

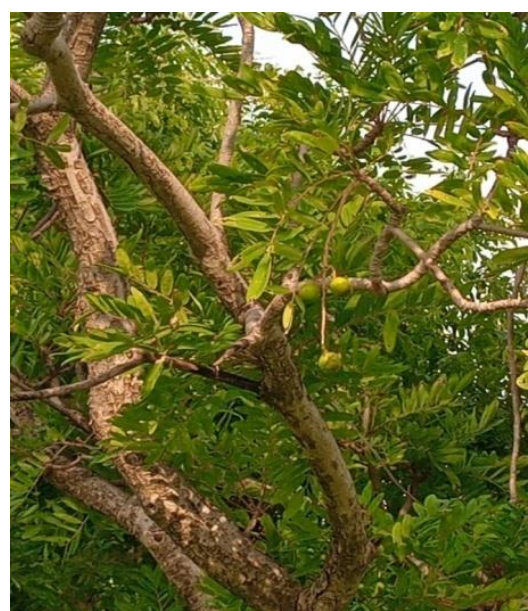

Haematostaphis barteri Hook. f.

A. Photographs of some most important resource species of the area of Zamay Forest Reserve

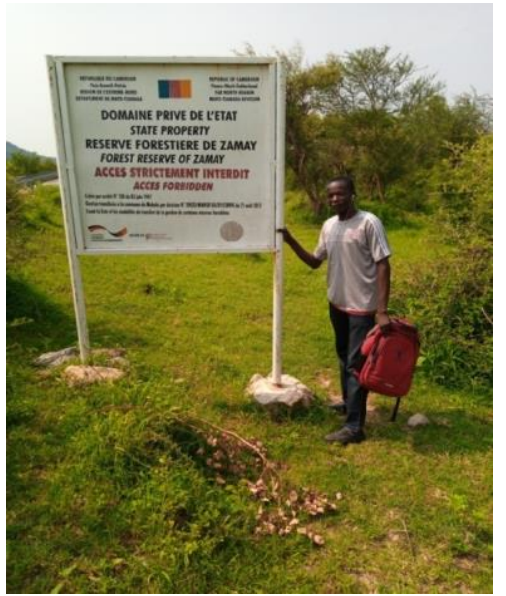

Indication plate of Zamay Forest Reserve

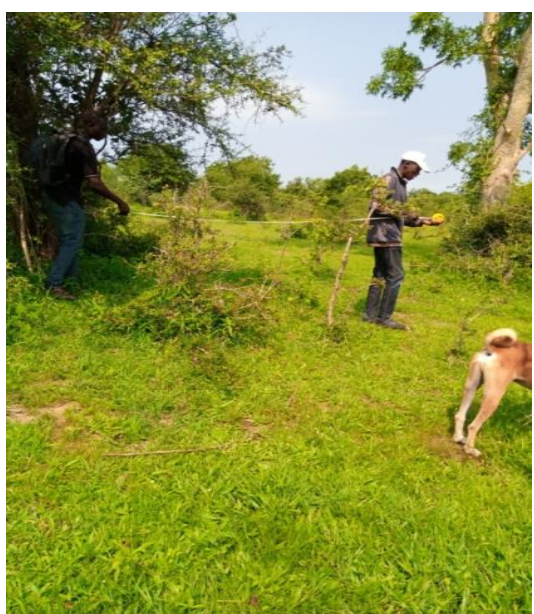

Establishment of the plots in field

B. Relevant photographs of the field 